\title{
Exploring Cultural Similarity and Cultural Diversity: A Cross-National Study of Nine Countries
}

\author{
Nancy J. Karlin ${ }^{1 *}$ and Joyce Weil ${ }^{2}$ \\ ${ }^{1}$ School of Psychological Sciences McKee 16d University of Northern Colorado, Greeley, CO -80639, USA; ${ }^{2}$ Gerontology Program, Gunter \\ Hall University of Northern Colorado, Greeley, CO-80639, USA
}

\begin{abstract}
Population aging is a universal, global issue with each country facing its own challenges to ensure the health of its older persons. We examined information gathered from 363 participants residing in nine societies (Japan, Saudi Arabia, China, Thailand, Botswana, Tunisia, U.S., South Africa, and Italy) about issues related to their self-reported aging experience. Using face-to-face interview and survey methods, information from older adults suggests evidence for both cultural similarity and diversity for these aging older adults. There is uniformity within the countries we studied in older adults' dislike of health changes and the experiencing financial concerns. Data collected also provides support for noticeable elements of diversity with regard to aging in each country and that divergent paths in aging exist. Findings point to recognition for cultural humility, recognizing the lack of knowledge for specific aging practices within individual societies, and a push toward cultural competence where more data is required to understand the true experiences within a group.
\end{abstract}

Keywords: Cultural context; Aging; Emic and etic constructs; Cultural diversity; Cross-national

\section{INTRODUCTION}

The world population is aging as reported by multiple researchers [1-5] using multiple sources (e.g., Population Reference Bureau; World Health Organization). This demographic trend is predicted to continue expanding the older age-cohorts globally $[5,6]$. The Valencia Forum coming out of the Second World Assembly on Ageing, Madrid, Spain in 2002 identified six research priorities to gaining a greater understanding of aging. One of the acknowledged research priorities was the need for additional examination into "quality of life and aging in diverse cultural, socio-economic and environmental situations" [7]. Recently, contemporary researchers have raised the discussion about the importance of examining aging using a cultural lens [8-14] and have made the international spotlight an integral part of the analysis on human aging. Crossnational investigations have become a central focus as scholars and practitioners give effort toward increasing knowledge about the aging experience.

When it comes to understanding the aging process much of the early data were obtained from residents within the United States. Numerous single country investigations followed that also focused on a specific age-related issue [15-19]. The current study expands upon existing research using single-location data that often cannot be generalized from one culture to another. It adds to the body of research by identifying emic (cultural diversity) and etic (cultural similarity) aging perceptions using multiple data collection sites. These assessments are relevant in developing a more comprehensive perspective of the aging experience using a multi-site, cross-cultural lens. This project is the culmination of data collection in nine different country locations. It is an attempt for the continued movement away from the sampling of middle-class European Americans or using only single country sites. Sites selected from four continents include both elder heavy and elder light countries.

\section{Cultural similarity and diversity}

Previous studies suggest the potential for both cultural uniformity and cultural diversity on various perceptions of aging. In terms of cultural similarity, or the approach to look for only communities across geographic settings, studies using multiple countries have produced findings that tend to focus on one issue and/or one region of the world. Examples include topics such as childhood socioeconomic status and late-adulthood mental health [20] mental health in European countries [21]; age differences in level of trust [22] psychological concerns and fear of losses [23].

${ }^{*}$ Correspondence to: Dr. Nancy J. Karlin, Professor, School of Psychological Sciences McKee 16d University of Northern Colorado, Greeley, CO -80639, USA, Tel: (970) 351-2717; E-mail: nancy.karlin@unco.edu

Received: May 03, 2019, Accepted: May 16, 2019, Published: May 23, 2019

Citation: Karlin NJ, Weil J (2019) Exploring Cultural Similarity and Cultural Diversity: A Cross-National Study of Nine Countries. J Aging Sci 7: 204.doi:10.35248/2329-8847.19.07.204

Copyright: (c) 2019 Karlin NJ, et al. This is an open-access article distributed under the terms of the Creative Commons Attribution License, which permits unrestricted use, distribution, and reproduction in any medium, provided the original author and source are credited. 
Etic or universal constructs identified from these studies point to changing age-related demographics along with concerns about personal aging (e.g., health care; well-being; social support; functional status, etc.). Universally, population aging as reported by multiple researchers [24] using multiple sources (e.g., Population Reference Bureau; World Health Organization) including country specific bureau of statistics. Current reported population numbers indicate an increasing older demographic along with predictions for an expanding older cohort $[5,6]$. The demography of aging is a common reference point cited in publications demonstrating the importance of demographic transition as the number of older adults' increases worldwide.

For every report of cultural similarity (etic constructs) there are just as many demonstrated paths of cultural diversity in aging, or ways that aging differs by one's culture. In other words, "observed differences between cultures may be the result of differences in features of culture" [24]. Examples of cultural diversity (emic constructs) report findings that center on gender and intergenerational co-residence as found in Egypt and Tunisia [25]. In some cultures, rural older adults do better with functional capacity and self-evaluation of health than urban older adults [26]. Older adults indicate similar concerns with regard to aging, but not everyone over the age of 65 reports the same exact type of concerns [27]. Research suggests that cultural diversity or emic constructs also exist for country specific perceptions of aging [28]. These authors assert substantial differences exist in cultures as to the preparation that occurs for old age and that practices are culture specific differing based on the "country, domain, and age group". In summary, review of the literature suggests limited consistency exists in variables or concepts used to understand the aging experience within a culture specific framework.

\section{Collectivist and individualist cultures}

Another way to study cultures and aging is to categorize them as individualistic or collective. Western cultures which are often recognized for their individualistic ideals [29]. Although seen as antithetical to collectivist cultures individualistic cultures place greater emphasis on competition where collectivist cultures center more on cooperation [30]. Collectivist countries recognize the interdependence found between people; whereas individualistic countries focus on self-actualization of goal obtainment. Published findings repeatedly indicate results look very different coming from countries seen as traditionally individualist versus those recognized as collectivist in orientation [31-35]. However, not all research identifies differences, but similarities between countries. Wacker and Roberto, Chan et al. [36,37] report that the level of positive association between trust influenced factors like: self-rated health, happiness, life satisfaction and quality of life for adults aged 50 and over from China, Ghana, India, Mexico, South Africa, and Russia. The authors also point out that their multinational findings are consistent with findings from Western countries. Of particular interest, Oksuzyan et al. [27] indicate similar concerns exist for older adults as reported by younger adult, however, not everyone over the age of 65 reports the same exact type of concerns. Therefore, although differences have been reported by prior studies with regard to aging similarity also exists.

\section{The current study}

The purpose of the study was to assess the common and unique components of aging in nine countries. The countries selected differ in their cultural practices, government policies, and other societal factors. Although prior research has identified various elements of aging within a specified culture, information on the overall aging experience across countries remains limited. Thus, we sought to examine the reported cultural uniformity and/ or diversity for experiences of aging (i.e., family dynamics, daily activities, perceptions of personal health and happiness, financial aspects of retirement, satisfaction with life, feelings/benefits of age, dislikes about age, looking forward to).

\section{METHODS}

Data were collected from four continents (North America, Asia, Africa, and Europe), from 2011 to 2018. Intentional efforts were taken to collect data from two or more locations in Asia, Africa, and Europe so as to obtain a broader understanding of aging outside the United States.

\section{Participants}

Participants resided in (in order of data collection) Italy, the United States, Thailand, Botswana, South Africa, Saudi Arabia, Tunisia, Japan, and China. Every attempt was made to obtain equal numbers of male to female participants (201 females; 160 males; 2 no report). The nine countries selected vary in whether the culture is primarily collectivist or individualist (Hofstede Insights, 2019). Generally considered to be collectivist cultures included samples from Japan ( $\mathrm{N}=23)$, Saudi Arabia $(\mathrm{N}=59)$, China $(\mathrm{N}=39)$, Thailand $(\mathrm{N}=59)$, Botswana $(\mathrm{N}=25)$, Tunisia $(\mathrm{N}=61)$. Individualist cultures samples: U.S. $(\mathrm{N}=40)$, South Africa $(\mathrm{N}=30)$, and Italy $(\mathrm{N}=27)$.

\section{Procedures}

\section{Translation process}

In each country, two experts were part of translating the English version of the survey into the local language preferred by the older adults. Initially, one expert would translate a version from English to the language of the region to be investigated. We would then have a back translation completed from that respective language to English. If the two experts agreed the translations maintained conceptual equivalence the translated version was used.

\section{Interview methods}

Older adults were primarily interviewed individually via a face-toface semi-structured survey with the exception of data collection from China, which was via an app (WeChat). With the mode of data collection noted, data from China were included in the analyses. Interviewers utilized a snowball sampling method to locate participants. Interviews consisted of a combination of closed and open-ended questions and took place primarily in the residence of an individual participant, local cafes or social gathering places. Study procedures were approved by an institutional review board (IRB) prior to data collection and interviewees went through the informed consent process. 


\section{Measures}

Demographic information was obtained along with data from a 35-item questionnaire created by Wacker and Roberto [36] for their book, aging social policies: An international perspective. The data collection instrument had an international perspective that included social policies [36]. The survey items were based on daily experiences of aging with a focus on family history, daily activities, programs and services that assist older adults, and the individual experience of aging. Demographic information was also obtained. Variable breakdowns are provided in table descriptions.

\section{Analytic strategy}

Data was collected using a semi-structured survey with open and closed questions. We computed descriptive statistics, including Pearson Correlation Coefficients, followed by inferential tests (Analysis of Variance, ANOVA, Analysis of Covariance, ANCOVA). All statistical analyses were completed using SPSS Version 25 with a significant $\mathrm{p}$-value of $<0.05$.

\section{RESULTS}

\section{Sample descriptive statistics}

Descriptive and univariate analyses were completed on 363 adult participants, 50 years of age or older (Table 1 ).

Age is the age of the individual reported at the time of the interview. Male is used as the reference category for gender. Marriage status is defined by four categories (married, divorced, widowed, never married). Delineation of geographical area for birth was a dichotomous variable using urban (densely developed area) as the reference category. ANOVA with post hoc Bonferroni tests were used to compare the nine cultural groups/countries. Differences were significant except for the marriage status categories. Even though every attempt was made to obtain equal numbers of male to female participants and age of participants, there were significant differences on reported gender $[F(8,360)=3.89, p$ $<0.001]$ and age $[F(8,362)=15.43, p<0.001]$ for the nine cohorts. A larger number of males served as respondents for the Japan and Tunisia sort, while a much smaller number of male participants were obtained for South African. Post hoc comparisons indicate the mean age was significantly different between groups with the greatest variation found between Saudi Arabian $(\mathrm{M}=61.0, \mathrm{SD}=7.7)$ and Italian participants $(M=79.6, S D=6.7)$. Differences were also evident for urban versus rural place of birth $[F(8,354)=30.73$, $\mathrm{p}<0.001]$. All respondents for those residing in Botswana were born in rural regions of the country while all of those interviewed from Saudi Arabia were born in urban areas. Differences for number of siblings $[F(8,355)=10.78, \mathrm{p}<0.001]$, number of children [F $(8,349)=18.30, p<0.001]$, and number of grandchildren [F $(8$, $354)=14.00, p<0.001]$ were reported between the societal groups. The number of reported daily activities also differ between groups $[F(8,354)=30.96, p<0.001]$

\section{CORRELATIONS}

Pearson product-moment correlations and p-values for key study variables are presented in Table 2 .

All of the linear relationships would be considered weak. There were significant correlations between the reported number of daily activities for an average day and several variables including a positive correlation with satisfaction with life, $r=0.124, n=352$, $\mathrm{p}=0.02$; and positive correlation with feelings about their current age, $r=0.108, n=352, p=043$. Careful review of the correlation matrix indicates the higher the number of daily activities the less overall satisfaction with life and reduced feelings about being a current age. There was no correlation reported between the total number of activities for an average day and being happy and healthy. Being happy and healthy was however positively correlated with satisfaction with life, $r=0.350, n=349, p=0.001$; and negatively

Table 1: Descriptive statistics ( $\mathrm{N}=363)$.

\begin{tabular}{|c|c|c|c|c|c|c|c|c|c|c|c|c|c|c|c|c|c|c|c|}
\hline \multirow[t]{2}{*}{ Variables } & \multicolumn{2}{|c|}{ Italy $(\mathrm{N}=27)$} & \multicolumn{2}{|c|}{ US $(\mathrm{N}=40)$} & \multicolumn{2}{|c|}{$\begin{array}{l}\text { Thailand } \\
(\mathrm{N}=59)\end{array}$} & \multicolumn{2}{|c|}{$\begin{array}{l}\text { Botswana } \\
(\mathrm{N}=25)\end{array}$} & \multicolumn{2}{|c|}{$\begin{array}{l}\text { South Africa } \\
\qquad(\mathrm{N}=30)\end{array}$} & \multicolumn{2}{|c|}{$\begin{array}{l}\text { Saudi Arabia } \\
\qquad(\mathrm{N}=59)\end{array}$} & \multicolumn{2}{|c|}{$\begin{array}{l}\text { Tunisia } \\
(\mathrm{N}=61)\end{array}$} & \multicolumn{2}{|c|}{$\begin{array}{l}\text { Japan } \\
(\mathrm{N}=23)\end{array}$} & \multicolumn{2}{|c|}{$\begin{array}{l}\text { China } \\
(\mathrm{N}=39)\end{array}$} & \multirow[t]{2}{*}{ p-value } \\
\hline & $\mathrm{N}(\%)$ & SD & $\mathrm{N}(\%)$ & SD & $\mathrm{N}(\%)$ & $\mathrm{SD}$ & $\mathrm{N}(\%)$ & SD & $\mathrm{N}(\%)$ & SD & $\mathrm{N}(\%)$ & SD & $\mathrm{N}$ & SD & $\mathrm{N}(\%)$ & SD & $\mathrm{N}(\%)$ & SD & \\
\hline Male & $\begin{array}{c}10 \\
(37)\end{array}$ & 0.49 & $\begin{array}{c}13 \\
(32)\end{array}$ & 0.47 & $\begin{array}{c}23 \\
(39)\end{array}$ & 0.49 & $\begin{array}{c}11 \\
(44)\end{array}$ & 0.5 & $\begin{array}{c}5 \\
(17)\end{array}$ & 0.37 & $\begin{array}{c}27 \\
(46)\end{array}$ & 0.5 & $\begin{array}{c}38 \\
(62)\end{array}$ & 0.48 & $\begin{array}{c}17 \\
(74)\end{array}$ & 0.44 & $\begin{array}{c}16 \\
(41)\end{array}$ & 0.49 & $.001^{* * *}$ \\
\hline Married & $\begin{array}{c}22 \\
(81)\end{array}$ & 0.42 & $\begin{array}{l}35 \\
(87)\end{array}$ & 0.35 & $\begin{array}{l}36 \\
(61)\end{array}$ & 0.58 & $\begin{array}{l}23 \\
(92)\end{array}$ & 0.27 & $\begin{array}{l}29 \\
(97)\end{array}$ & 0.18 & $\begin{array}{l}56 \\
(95)\end{array}$ & 0.32 & $\begin{array}{c}57 \\
(93)\end{array}$ & 0.25 & $\begin{array}{c}17 \\
(74)\end{array}$ & 0.73 & $\begin{array}{c}39 \\
(100)\end{array}$ & 0 & 0.75 \\
\hline Divorced & 0 & - & 0 & - & 0 & - & 0 & - & 0 & - & 1 & - & 0 & - & 2 & 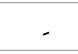 & 0 & - & - \\
\hline Widowed & 4 & - & 4 & - & 10 & - & 0 & - & 0 & - & 1 & - & 2 & - & 0 & - & 0 & - & - \\
\hline Never married & 1 & - & 1 & - & 8 & - & 2 & - & 1 & - & 1 & - & 2 & - & 4 & - & 0 & - & - \\
\hline Born Urban & $\begin{array}{c}10 \\
(37)\end{array}$ & 0.49 & $\begin{array}{c}13 \\
(32)\end{array}$ & 0.48 & $\begin{array}{c}21 \\
(36)\end{array}$ & 0.48 & 0 & 0 & $\begin{array}{c}14 \\
(47)\end{array}$ & 0.5 & $\begin{array}{c}59 \\
(100)\end{array}$ & 0 & $\begin{array}{c}44 \\
(72)\end{array}$ & 0.45 & $\begin{array}{c}21 \\
(91)\end{array}$ & 0.21 & $\begin{array}{c}38 \\
(97)\end{array}$ & 0.16 & $.001^{* \star *}$ \\
\hline Variables & Mean & SD & Mean & SD & Mean & SD & Mean & SD & Mean & SD & Mean & SD & Mean & SD & Mean & SD & Mean & SD & p-value \\
\hline Age & 79.6 & 6.7 & 72.7 & 9 & 68.6 & 6.2 & 71.9 & 7.9 & 71.7 & 7.1 & 61 & 7.7 & 70 & 7.2 & 75.4 & 7.4 & 69.7 & 8.5 & $.001^{* \star *}$ \\
\hline " of Siblings & 4.3 & 2.9 & 3.8 & 3.2 & 5.4 & 2.3 & 7.5 & 2.6 & 6.1 & 2.8 & 6.9 & 3.4 & 5 & 2.5 & 2.3 & 2.7 & 3.7 & 1.9 & $.001^{* * t}$ \\
\hline " of Children & 2.4 & 0.98 & 2.6 & 1.2 & 3.1 & 1.6 & 6 & 1.9 & 4.7 & 2.5 & 4 & 2.1 & 3.3 & 1.9 & 1.6 & 1.1 & 1.8 & 1.2 & $.001^{* * *}$ \\
\hline $\begin{array}{c}\text { \# of } \\
\text { Grandchildren }\end{array}$ & 3 & 2.3 & 5.2 & 5.3 & 4.6 & 5.9 & 11.7 & 7.5 & 8.3 & 5.2 & 3.3 & 5.2 & 2.8 & 3.8 & 1.3 & 1.7 & 1.7 & 1.7 & $.001^{* * *}$ \\
\hline $\begin{array}{c}\text { Number of } \\
\text { Daily Activities }\end{array}$ & 3 & 1.3 & 4.3 & 2 & 1.3 & 0.95 & 1.9 & 0.97 & 2.5 & 1.5 & 3.3 & 1.6 & 5.3 & 2 & 6 & 3 & 3.6 & 1.8 & $.001^{* \star *}$ \\
\hline
\end{tabular}

Note. ${ }^{* * *} / * *$ indicate significant differences at $0.05 / 0.01 / 0.001$ levels based on group mean comparisons between samples 
Table 2: Correlation matrix.

\begin{tabular}{|c|c|c|c|c|c|c|}
\hline Variables & 1 & 2 & 3 & 4 & 5 & 6 \\
\hline Daily Activity Level & 1 & - & - & - & - & - \\
\hline Happy/Healthy & 0.03 & 1 & - & - & - & - \\
\hline Satisfaction with Life & $0.124^{*}$ & $0.350^{\star *}$ & 1 & - & - & - \\
\hline Retirement Benefits & -0.024 & $-0.140^{* *}$ & -0.085 & 1 & - & - \\
\hline Benefits Sufficient & 0.083 & -0.068 & $-0.176^{* *}$ & 0.09 & 1 & - \\
\hline Feelings about age & $0.108^{*}$ & 0.026 & 0.054 & -0.086 & 0.053 & 1 \\
\hline
\end{tabular}

Notes: Daily Activity Level is the total number of activities for an average day; Happy/Healthy is no (0) or yes (1); Satisfaction with life ranges from 1 (very) to 4 (not very); Retirement benefits are no (0) or yes (1); Benefits sufficient is no (0) or yes (1); Feelings about age ranges from (1) well to 4 (not well); Two-tailed significance ${ }^{*} \mathrm{p}<0.05,{ }^{* *} \mathrm{p}<0.01$;

correlated with receiving retirement benefits/pension, $r=-0.140$, $n=342, p=0.009$. Thus, the happy and healthy an individual is the lower the overall satisfaction with life and the less likely they were be receiving retirement benefits. Although these findings may not make intuitive sense more discernment is evident when considered with the number of individuals who reported still working even after a formal retirement age $(4.7 \%)$ along with most who said no to retirement benefits being available had not applied for benefits that might be waiting to be claimed (7.4\%). Satisfaction with life was found to be negatively correlated with whether retirement benefits are sufficient, $\mathrm{r}=-0.176, \mathrm{n}=275, \mathrm{p}=0.003$. Those who state not being very satisfied with life report benefits received as not sufficient. Feelings about being his or her current age were not correlated with being happy or healthy, overall satisfaction with life, and whether retirement benefits were available or sufficient.

\section{Frequencies and group mean comparisons}

Frequencies and group mean comparisons of key study variables are provided in Table 3.

ANOVA signals significant differences between countries for each of the variables. For being happy and health Italy reported the lowest percentage $(48.2 \%)$ with China $(100 \%)$ as the highest closely followed by Saudi Arabia (93.2\%) and Japan (91.3\%)[F(8, $344)=10.20, p<0.001]$. The highest overall satisfaction with life came from Saudi participants $(M=1.17$, range $1-4)$ followed by the U.S. $(M=1.33)$, South Africa $(M=1.62)$, Japan, $(M=1.70)$, Thailand $(\mathrm{M}=1.71)$, China $(\mathrm{M}=1.76)$, Botswana $(\mathrm{M}=1.84)$, Italy $(\mathrm{M}=1.85)$, and Tunisia $(M=1.92)[F(8,351)=16.97, \mathrm{p}<0.001]$. More participants from China and the U.S. reported having retirement benefits $[\mathrm{F}(8$, $344)=4.12, p<0.001$ ]; however, the sources of benefits were broad for U.S. respondents where support came from either a pension or government source for Chinese participants $[\mathrm{F}(8,313)=26.55$, $\mathrm{p}<0.001]$. The data indicates the reported sufficiency of retirement benefits varied between groups $[F(8,282)=5.78, p<0.001]$. The two questions that drew the lowest response rate within some groupings were whether extra revenue was available $[F(8,317)=18.18, p<0.001]$ and whether services were not covered by retirement or personal resources $[F(6,221)=14.02, p<0.001]$ and should be interpreted with caution. Both variables touch on the financial situation of the individual and may impact a willingness to respond to a specific type of question. It appears that cultural context plays a role in what is reported as the most important benefit of a respondent's current age $[F(8,317)=18.18, p<0.001]$. Although some variation is evident what participants across country dislike most about getting older is the change in overall health $[F(8,330)=6.50, p<0.001]$. Even with the reported differences on what respondents were most often looking forward to being with family was indicated frequently [F (8, $346)=12.66$, $p<0.001$ ] with one exception. Botswanan participants most often stated they were looking forward to a peaceful death, which may be associated with having one of the highest death rates as a result of HIV/AIDS (WHO, 2017). Many of the older adults interviewed were caring for grandchildren while adult parents went to urban areas to obtain work or were deceased due to contracting HIV/AIDS. Also noted was a broad range of advice provided to the youth of each respective country with variations evident between groups $[F(8,334)=27.18, \mathrm{p}<0.001]$.

To examine whether respondents by country differed on responses for the study variables of interest, we computed ANCOVAs with age and gender as the covariates. Separate analyses were performed for the variables of interest. There was a significant difference in being happy and healthy $\left[F(8,344)=9.53, p<0.001, \eta p^{2}=0.18\right]$ between the countries whilst adjusting for age and gender with a small effect size. For satisfaction with life a significant difference was also evident $\left[F(8,339)=4.97, p<0.001, \eta p^{2}=0.10\right]$. Similar results were found for retirement benefits $[F(8,332)=4.85, p<0.001$, $\left.\eta \mathrm{p}^{2}=0.10\right]$, where retirement support came from $[\mathrm{F}(8,301)=16.73$, $\left.\mathrm{p}<0.001, \eta \mathrm{p}^{2}=0.30\right]$, whether benefits are sufficient $[\mathrm{F}(8,270)=5.87$, $\left.\mathrm{p}<0.001, \eta \mathrm{p}^{2}=0.14\right]$, whether extra revenue was available $[\mathrm{F}(8$, $305)=5.90, \mathrm{p}<0.001, \eta \mathrm{p}^{2}=0.13$ ], whether services are not covered by retirement or personal resources $[F(6,211)=16.73, p<0.001$, $\left.\eta p^{2}=0.27\right]$, how they feel about their current age $[F(8,339)=2.14$, $\left.\mathrm{p}<0.03, \eta \mathrm{p}^{2}=0.04\right]$, benefits of current age $[F(8,327)=2.44, \mathrm{p}<0.01$, $\left.\eta \mathrm{p}^{2}=0.05\right]$, what participants dislike about being his or her current age $\left[\mathrm{F}(8,319)=2.43, \mathrm{p}<0.01, \eta \mathrm{p}^{2}=0.05\right]$, what they are most looking forward to in retirement $\left[F(8,334)=4.83, p<0.001, \eta p^{2}=0.10\right]$, and daily activity level $\left[\mathrm{F}(8,350)=30.30, \mathrm{p}<0.001, \eta \mathrm{p}^{2}=0.40\right]$.

\section{Testing individualistic vs. collectivist category differences}

In an effort to identify possible cultural issues that influence the aging experience, mean comparisons between the collectivist and individualistic cultures using ANOVA were completed. Results indicate that divergent paths to aging exist. An ANOVA with culture-type (collectivist or individualistic) as the independent variable was run for variables of interest as dependent variables. For the cultural component a significant effect was found for being happy and healthy, $[F(1,356)=4.18, p<0.04]$ indicating those residing in individualistic cultures reported higher levels $(\mathrm{M}=1.37$, $\mathrm{SD}=1.67)$ than those interviewed from collectivist cultures $(\mathrm{M}=1.95, \mathrm{SD}=2.58)$. We computed one-way ANOVAs comparing the cultural component for satisfaction with life, whether 
Table 3: Frequencies of key study variables $(\mathrm{N}=363)$.

\begin{tabular}{|c|c|c|c|c|c|c|c|c|c|c|c|c|c|c|c|c|c|c|c|c|}
\hline \multirow[t]{2}{*}{ Variables } & & \multicolumn{2}{|c|}{ Italy $(\mathrm{N}=27)$} & \multicolumn{2}{|c|}{ U.S. $(\mathrm{N}=40)$} & \multicolumn{2}{|c|}{$\begin{array}{l}\text { Thailand } \\
(\mathrm{N}=59)\end{array}$} & \multicolumn{2}{|c|}{$\begin{array}{c}\text { Botswana } \\
(\mathrm{N}=25)\end{array}$} & \multicolumn{2}{|c|}{$\begin{array}{l}\text { South Africa } \\
\qquad(\mathrm{N}=30)\end{array}$} & \multicolumn{2}{|c|}{$\begin{array}{l}\text { Saudi Arabia } \\
\qquad(\mathrm{N}=59)\end{array}$} & \multicolumn{2}{|c|}{$\begin{array}{l}\text { Tunisia } \\
(\mathrm{N}=61)\end{array}$} & \multicolumn{2}{|c|}{$\begin{array}{l}\text { Japan } \\
(\mathrm{N}=23)\end{array}$} & \multicolumn{2}{|c|}{$\begin{array}{l}\text { China } \\
(\mathrm{N}=39)\end{array}$} & \multirow[t]{2}{*}{$\mathrm{p}$-value } \\
\hline & & $\mathrm{N}(\%)$ & SD & $\mathrm{N}(\%)$ & SD & $\mathrm{N}(\%)$ & SD & $\mathrm{N}(\%)$ & SD & $\mathrm{N}(\%)$ & SD & $\mathrm{N}(\%)$ & $\mathrm{SD}$ & $\mathrm{N}$ & $\mathrm{SD}$ & $\mathrm{N}(\%)$ & SD & $\mathrm{N}(\%)$ & $\mathrm{SD}$ & \\
\hline Happy/Healthy & (Yes) & $\begin{array}{c}13 \\
(48.2)\end{array}$ & 2.5 & $\begin{array}{c}36 \\
(90.0)\end{array}$ & 1.3 & $\begin{array}{c}50 \\
(84.7)\end{array}$ & 1.8 & $\begin{array}{c}18 \\
(72.0)\end{array}$ & 3 & $\begin{array}{c}25 \\
(83.3)\end{array}$ & 0.37 & $\begin{array}{c}55 \\
(93.2)\end{array}$ & 0.42 & $\begin{array}{c}36 \\
(59.0)\end{array}$ & 4 & $\begin{array}{c}21 \\
(91.3)\end{array}$ & 0.66 & $\begin{array}{c}39 \\
(100)\end{array}$ & 0 & $0.001^{* *+\cdots}$ \\
\hline \multicolumn{21}{|c|}{ Satisfaction with Life } \\
\hline Very & & $\begin{array}{c}13 \\
(48.1)\end{array}$ & 0.95 & $\begin{array}{c}26 \\
(65.0)\end{array}$ & 0.47 & $\begin{array}{c}25 \\
(42.4)\end{array}$ & 0.77 & $\begin{array}{c}8 \\
(32.0)\end{array}$ & 0.83 & $\begin{array}{c}14 \\
(46.7)\end{array}$ & 0.72 & $\begin{array}{c}52 \\
(88.1)\end{array}$ & 0.59 & $\begin{array}{c}13 \\
(21.3)\end{array}$ & 0.64 & $\begin{array}{c}11 \\
(47.8)\end{array}$ & 0.76 & $\begin{array}{c}12 \\
(30.8)\end{array}$ & 0.63 & $0.001^{s+n}$ \\
\hline Fairly & & $\begin{array}{c}6 \\
(22.2)\end{array}$ & - & $\begin{array}{c}13 \\
(32.5)\end{array}$ & - & $\begin{array}{c}28 \\
(47.5)\end{array}$ & & $\begin{array}{c}6 \\
(24.0)\end{array}$ & - & $\begin{array}{c}13 \\
(43.3)\end{array}$ & - & $\begin{array}{c}4 \\
(6.8)\end{array}$ & - & $\begin{array}{c}42 \\
(68.9)\end{array}$ & - & $\begin{array}{c}8 \\
(34.8)\end{array}$ & - & $\begin{array}{c}24 \\
(61.5)\end{array}$ & & - \\
\hline A little & & $\begin{array}{c}7 \\
(25.9)\end{array}$ & - & $\begin{array}{c}0 \\
(00.0)\end{array}$ & - & $\begin{array}{c}2 \\
(3.4)\end{array}$ & - & $\begin{array}{c}5 \\
(20.0)\end{array}$ & - & $\begin{array}{c}1 \\
(3.3)\end{array}$ & - & $\begin{array}{c}0 \\
(00.0)\end{array}$ & - & $\begin{array}{c}4 \\
(6.6)\end{array}$ & - & $\begin{array}{c}4 \\
(17.4)\end{array}$ & - & $\begin{array}{c}1 \\
(2.6)\end{array}$ & - & - \\
\hline Not Very & & $\begin{array}{c}1 \\
(3.7)\end{array}$ & - & $\begin{array}{c}0 \\
(00.0)\end{array}$ & - & $\begin{array}{c}3 \\
(5.1)\end{array}$ & - & $\begin{array}{c}0 \\
(00.0)\end{array}$ & - & $\begin{array}{c}1 \\
(3.3)\end{array}$ & - & $\begin{array}{c}2 \\
(3.4)\end{array}$ & - & $\begin{array}{c}2 \\
(3.3)\end{array}$ & - & $\begin{array}{c}0 \\
(00.0)\end{array}$ & - & $\begin{array}{c}1 \\
(2.6)\end{array}$ & - & - \\
\hline Retirement Benefits & (Yes) & $\begin{array}{c}24 \\
(88.9)\end{array}$ & 0.32 & $\begin{array}{c}30 \\
(75.0)\end{array}$ & 0.35 & $\begin{array}{c}57 \\
(96.6)\end{array}$ & 0.18 & $\begin{array}{c}22 \\
(88.0)\end{array}$ & 0.33 & $\begin{array}{c}27 \\
(90.0)\end{array}$ & 0.3 & $\begin{array}{c}36 \\
(61.0)\end{array}$ & 0.48 & $\begin{array}{c}49 \\
(80.3)\end{array}$ & 0.4 & $\begin{array}{c}18 \\
(78.3)\end{array}$ & 0.47 & $\begin{array}{c}0 \\
(00.0)\end{array}$ & 0 & $0.001^{s+n}$ \\
\hline Not Retired & & $\begin{array}{c}0 \\
(00.0)\end{array}$ & - & $\begin{array}{c}5 \\
(12.5)\end{array}$ & - & $\begin{array}{c}0 \\
(00.0)\end{array}$ & - & $\begin{array}{c}3 \\
(12.0)\end{array}$ & & $\begin{array}{c}0 \\
(00.0)\end{array}$ & - & $\begin{array}{c}6 \\
(10.2)\end{array}$ & - & $\begin{array}{c}0 \\
(00.0)\end{array}$ & - & $\begin{array}{c}3 \\
(13.0)\end{array}$ & - & $\begin{array}{c}38 \\
(97.4)\end{array}$ & - & - \\
\hline \multicolumn{21}{|c|}{ Source of Benefits } \\
\hline Spouse Pension & & $\begin{array}{c}6 \\
(22.0)\end{array}$ & 1.7 & $\begin{array}{c}3 \\
(7.5)\end{array}$ & 3.2 & $\begin{array}{c}0 \\
(00 .)\end{array}$ & 3.4 & $\begin{array}{c}25 \\
(100)\end{array}$ & 0 & $\begin{array}{c}1 \\
(3.3)\end{array}$ & 2.8 & $\begin{array}{c}3 \\
(5.1)\end{array}$ & 2.5 & $\begin{array}{c}6 \\
(9.8)\end{array}$ & 3.6 & $\begin{array}{c}1 \\
(4.3)\end{array}$ & 0.53 & $\begin{array}{c}0 \\
(00.0)\end{array}$ & 3 & $0.001^{* *+1}$ \\
\hline Pension & & $\begin{array}{c}15 \\
(55.6)\end{array}$ & - & $\begin{array}{c}13 \\
(32.5)\end{array}$ & - & $\begin{array}{c}29 \\
(49.2)\end{array}$ & - & $\begin{array}{c}0 \\
(00.0)\end{array}$ & - & $\begin{array}{c}4 \\
(13.3)\end{array}$ & - & $\begin{array}{c}3 \\
(5.1)\end{array}$ & - & $\begin{array}{c}19 \\
(31.1)\end{array}$ & - & $\begin{array}{c}16 \\
(69.6)\end{array}$ & - & $\begin{array}{c}9 \\
(23.1)\end{array}$ & - & - \\
\hline $\begin{array}{l}\text { Pension \& Spouse } \\
\text { Pension }\end{array}$ & & $\begin{array}{c}1 \\
(3.7)\end{array}$ & - & $\begin{array}{c}5 \\
(12.5)\end{array}$ & - & $\begin{array}{c}1 \\
(1.7)\end{array}$ & - & $\begin{array}{c}0 \\
(00.0)\end{array}$ & - & $\begin{array}{c}0 \\
(00.0)\end{array}$ & - & $\begin{array}{c}0 \\
(00.0)\end{array}$ & - & $\begin{array}{c}1 \\
(1.6)\end{array}$ & - & $\begin{array}{c}1 \\
(4.3)\end{array}$ & - & $\begin{array}{c}0 \\
(00.0)\end{array}$ & - & - \\
\hline Disability/Other & & $\begin{array}{c}2 \\
(7.4)\end{array}$ & - & $\begin{array}{c}0 \\
(00.0)\end{array}$ & - & $\begin{array}{c}2 \\
(3.4)\end{array}$ & - & $\begin{array}{c}0 \\
(00.0)\end{array}$ & - & $\begin{array}{c}1 \\
(3.3)\end{array}$ & - & $\begin{array}{c}0 \\
(00.0)\end{array}$ & - & $\begin{array}{c}1 \\
(1.6)\end{array}$ & - & $\begin{array}{c}0 \\
(00.0)\end{array}$ & . & $\begin{array}{c}0 \\
(00.0)\end{array}$ & - & - \\
\hline $\begin{array}{l}\text { Government Only } \\
\text { Pension }\end{array}$ & & $\begin{array}{c}1 \\
(3.7)\end{array}$ & - & $\begin{array}{c}10 \\
(25.0)\end{array}$ & - & $\begin{array}{c}25 \\
(42.4)\end{array}$ & - & $\begin{array}{c}0 \\
(00.0)\end{array}$ & - & $\begin{array}{c}18 \\
(60.0)\end{array}$ & - & $\begin{array}{c}32 \\
(54.2)\end{array}$ & - & $\begin{array}{c}20 \\
(32.8)\end{array}$ & - & $\begin{array}{c}0 \\
(00.0)\end{array}$ & - & $\begin{array}{c}29 \\
(74.4)\end{array}$ & 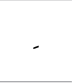 & - \\
\hline No Response & & $\begin{array}{c}2 \\
(7.4)\end{array}$ & - & $\begin{array}{c}9 \\
(22.5)\end{array}$ & - & $\begin{array}{c}2 \\
(3.4)\end{array}$ & - & $\begin{array}{c}0 \\
(00.0)\end{array}$ & - & $\begin{array}{c}3 \\
(10.0)\end{array}$ & - & $\begin{array}{c}13 \\
(22.0)\end{array}$ & - & $\begin{array}{c}14 \\
(23.0)\end{array}$ & - & $\begin{array}{c}5 \\
(21.7)\end{array}$ & - & $\begin{array}{c}1 \\
(2.6)\end{array}$ & 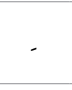 & - \\
\hline Benefits Sufficient & (Yes) & $\begin{array}{c}8 \\
(29.6)\end{array}$ & 0.87 & $\begin{array}{c}23 \\
(57.5)\end{array}$ & 0.89 & $\begin{array}{c}22 \\
(37.3)\end{array}$ & 0.47 & $\begin{array}{c}1 \\
(4.0)\end{array}$ & 0.98 & $\begin{array}{c}3 \\
(10.0)\end{array}$ & 0.31 & $\begin{array}{c}38 \\
(64.4)\end{array}$ & 0.94 & $\begin{array}{c}16 \\
(26.2)\end{array}$ & 0.9 & $\begin{array}{c}13 \\
(56.5)\end{array}$ & 1 & $\begin{array}{c}27 \\
(69.2)\end{array}$ & 0.45 & $0.001^{\ldots+\cdots}$ \\
\hline Somewhat & & $\begin{array}{c}1 \\
(3.7)\end{array}$ & - & $\begin{array}{c}2 \\
(5.0)\end{array}$ & - & $\begin{array}{c}0 \\
(00.0)\end{array}$ & - & $\begin{array}{c}1 \\
(4.0)\end{array}$ & - & $\begin{array}{c}0 \\
(00.0)\end{array}$ & - & $\begin{array}{c}4 \\
(6.8)\end{array}$ & - & $\begin{array}{c}2 \\
(3.3)\end{array}$ & - & $\begin{array}{c}2 \\
(8.7)\end{array}$ & - & $\begin{array}{c}0 \\
(00.0)\end{array}$ & - & - \\
\hline Extra Revenue & (Yes) & $\begin{array}{c}3 \\
(11.1)\end{array}$ & 0.32 & $\begin{array}{c}24 \\
(60.0)\end{array}$ & 0.44 & $\begin{array}{c}38 \\
(64.4)\end{array}$ & 0 & $\begin{array}{c}13 \\
(52.0)\end{array}$ & 0.49 & $\begin{array}{c}9 \\
(30.0)\end{array}$ & 0.68 & $\begin{array}{c}36 \\
(61.0)\end{array}$ & 0.48 & $\begin{array}{c}24 \\
(39.3)\end{array}$ & 0.77 & $\begin{array}{c}10 \\
(43.5)\end{array}$ & 0.51 & $\begin{array}{c}20 \\
(51.3)\end{array}$ & 0.5 & $0.001^{* * *}$ \\
\hline No & & $\begin{array}{c}24 \\
(88.9)\end{array}$ & - & $\begin{array}{c}8 \\
(20.0)\end{array}$ & - & $\begin{array}{c}0 \\
(00.0)\end{array}$ & - & $\begin{array}{c}8 \\
(32.0)\end{array}$ & - & $\begin{array}{c}19 \\
(63.3)\end{array}$ & - & $\begin{array}{c}19 \\
(32.2)\end{array}$ & - & $\begin{array}{c}34 \\
(55.7)\end{array}$ & - & $\begin{array}{c}9 \\
(39.1)\end{array}$ & - & $\begin{array}{c}18 \\
(46.2)\end{array}$ & - & 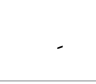 \\
\hline Services Not Covered & (Yes) & $\begin{array}{c}15 \\
(55.6)\end{array}$ & 0 & $\begin{array}{c}18 \\
(45.0)\end{array}$ & 0.48 & - & - & - & - & $\begin{array}{c}5 \\
(16.7)\end{array}$ & 0.43 & $\begin{array}{c}17 \\
(28.8)\end{array}$ & 0.47 & $\begin{array}{c}12 \\
(19.6)\end{array}$ & 0.5 & $\begin{array}{c}13 \\
(56.5)\end{array}$ & 0.5 & $\begin{array}{c}36 \\
(92.3)\end{array}$ & 0.27 & $0.001^{*+*}$ \\
\hline No Response & & $\begin{array}{c}12 \\
(44.4)\end{array}$ & - & $\begin{array}{c}12 \\
(30.0)\end{array}$ & - & $\begin{array}{c}59 \\
(100)\end{array}$ & - & $\begin{array}{c}25 \\
(100)\end{array}$ & . & $\begin{array}{c}9 \\
(30.0)\end{array}$ & . & $\begin{array}{c}9 \\
(15.3)\end{array}$ & - & $\begin{array}{c}15 \\
(24.9)\end{array}$ & - & $\begin{array}{c}0 \\
(00.0)\end{array}$ & - & $\begin{array}{c}0 \\
(00.0)\end{array}$ & - & - \\
\hline \multicolumn{21}{|c|}{ Feelings About Age } \\
\hline Well & & $\begin{array}{c}15 \\
(55.6)\end{array}$ & 1.4 & $\begin{array}{c}8 \\
(20.0)\end{array}$ & 0.99 & $\begin{array}{c}15 \\
(25.4)\end{array}$ & 1.1 & $\begin{array}{c}6 \\
(24.0)\end{array}$ & 1.2 & $\begin{array}{c}9 \\
(30.0)\end{array}$ & 1.1 & $\begin{array}{c}21 \\
(35.6)\end{array}$ & 1.1 & $\begin{array}{c}25 \\
(41.0)\end{array}$ & 1.2 & $\begin{array}{c}1 \\
(4.3)\end{array}$ & 1.2 & $\begin{array}{c}3 \\
(7.7)\end{array}$ & 0.85 & $0.001^{* *+}$ \\
\hline Fine & & $\begin{array}{c}4 \\
(14.8)\end{array}$ & - & $\begin{array}{c}10 \\
(25.0)\end{array}$ & - & $\begin{array}{c}20 \\
(33.9)\end{array}$ & - & $\begin{array}{c}4 \\
(16.0)\end{array}$ & - & $\begin{array}{c}2 \\
(6.7)\end{array}$ & - & $\begin{array}{c}3 \\
(5.1)\end{array}$ & - & $\begin{array}{c}2 \\
(3.3)\end{array}$ & - & $\begin{array}{c}17 \\
(73.9)\end{array}$ & - & $\begin{array}{c}9 \\
(23.1)\end{array}$ & - & - \\
\hline Could be better & & $\begin{array}{c}0 \\
(00.0)\end{array}$ & - & $\begin{array}{c}14 \\
(35.0)\end{array}$ & - & $\begin{array}{c}2 \\
(3.4)\end{array}$ & - & $\begin{array}{c}4 \\
(16.0)\end{array}$ & - & $\begin{array}{c}11 \\
(36.7)\end{array}$ & . & $\begin{array}{c}20 \\
(33.9)\end{array}$ & - & $\begin{array}{c}15 \\
(24.6)\end{array}$ & - & $\begin{array}{c}3 \\
(13.0)\end{array}$ & - & $\begin{array}{c}21 \\
(53.8)\end{array}$ & - & - \\
\hline Not Well & & $\begin{array}{c}7 \\
(25.9)\end{array}$ & - & $\begin{array}{c}6 \\
(15.0)\end{array}$ & - & $\begin{array}{c}19 \\
(32.2)\end{array}$ & - & $\begin{array}{c}8 \\
(32.0)\end{array}$ & - & $\begin{array}{c}8 \\
(26.7)\end{array}$ & . & $\begin{array}{c}13 \\
(22.0)\end{array}$ & - & $\begin{array}{c}19 \\
(31.1)\end{array}$ & - & $\begin{array}{c}2 \\
(8.7)\end{array}$ & - & $\begin{array}{c}6 \\
(15.4)\end{array}$ & - & - \\
\hline \multicolumn{21}{|c|}{ Benefits of Age } \\
\hline $\begin{array}{c}\text { Enjoy Visiting with } \\
\text { People }\end{array}$ & & $\begin{array}{c}7 \\
(25.9) \\
\end{array}$ & 2.3 & $\begin{array}{c}6 \\
(15.0) \\
\end{array}$ & 4.6 & $\begin{array}{c}5 \\
(8.5) \\
\end{array}$ & 3.9 & $\begin{array}{c}6 \\
(24.0) \\
\end{array}$ & 5 & $\begin{array}{c}12 \\
(40.0)\end{array}$ & 6.6 & $\begin{array}{c}11 \\
(18.6) \\
\end{array}$ & 6.2 & $\begin{array}{c}16 \\
(26.2) \\
\end{array}$ & 4.6 & $\begin{array}{c}2 \\
(8.7) \\
\end{array}$ & 3.7 & $\begin{array}{c}5 \\
(12.8) \\
\end{array}$ & 4.4 & $0.001^{\cdots+}$ \\
\hline Everything & & $\begin{array}{c}4 \\
(14.8)\end{array}$ & - & $\begin{array}{c}1 \\
(2.5)\end{array}$ & - & $\begin{array}{c}0 \\
(00.0)\end{array}$ & . & $\begin{array}{c}0 \\
(00.0)\end{array}$ & - & $\begin{array}{c}1 \\
(3.3)\end{array}$ & - & $\begin{array}{c}1 \\
(1.7)\end{array}$ & . & $\begin{array}{c}0 \\
(00.0)\end{array}$ & - & $\begin{array}{c}0 \\
(00.0)\end{array}$ & . & $\begin{array}{c}6 \\
(15.4)\end{array}$ & - & - \\
\hline Experiences & & $\begin{array}{c}3 \\
(11.1)\end{array}$ & . & $\begin{array}{c}3 \\
(7.5)\end{array}$ & - & $\begin{array}{c}4 \\
(6.8)\end{array}$ & - & $\begin{array}{c}2 \\
(8.0)\end{array}$ & - & $\begin{array}{c}3 \\
(10.0)\end{array}$ & - & $\begin{array}{c}1 \\
(1.7)\end{array}$ & - & $\begin{array}{c}5 \\
(8.2)\end{array}$ & - & $\begin{array}{c}0 \\
(00.0)\end{array}$ & . & $\begin{array}{c}5 \\
(12.8)\end{array}$ & 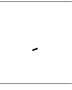 & - \\
\hline Health & & $\begin{array}{c}1 \\
(3.7)\end{array}$ & . & $\begin{array}{c}0 \\
(00.0)\end{array}$ & & $\begin{array}{c}3 \\
(5.1)\end{array}$ & & $\begin{array}{c}0 \\
(00.0)\end{array}$ & - & $\begin{array}{c}0 \\
(00.0)\end{array}$ & - & $\begin{array}{c}4 \\
(6.8)\end{array}$ & - & $\begin{array}{c}3 \\
(4.9)\end{array}$ & - & $\begin{array}{c}1 \\
(4.3)\end{array}$ & . & $\begin{array}{c}7 \\
(17.9)\end{array}$ & - & - \\
\hline
\end{tabular}




\begin{tabular}{|c|c|c|c|c|c|c|c|c|c|c|c|c|c|c|c|c|c|c|c|}
\hline Inner Peace & $\begin{array}{c}5 \\
(18.5)\end{array}$ & - & $\begin{array}{c}2 \\
(5.0)\end{array}$ & - & $\begin{array}{c}3 \\
(5.1)\end{array}$ & - & $\begin{array}{c}0 \\
(00.0)\end{array}$ & - & $\begin{array}{c}1 \\
(3.3)\end{array}$ & - & $\begin{array}{c}11 \\
(18.6)\end{array}$ & - & $\begin{array}{c}11 \\
(18.0)\end{array}$ & - & $\begin{array}{c}0 \\
(00.0)\end{array}$ & & $\begin{array}{c}2 \\
(5.1)\end{array}$ & - & - \\
\hline Nothing & $\begin{array}{c}4 \\
(14.8)\end{array}$ & $\cdot$ & $\begin{array}{c}2 \\
(5.0)\end{array}$ & - & $\begin{array}{c}2 \\
(3.4)\end{array}$ & & $\begin{array}{c}2 \\
(8.0)\end{array}$ & - & $\begin{array}{c}0 \\
(00.0)\end{array}$ & - & $\begin{array}{c}2 \\
(3.4)\end{array}$ & - & $\begin{array}{c}5 \\
(8.2)\end{array}$ & - & $\begin{array}{c}13 \\
(56.5)\end{array}$ & & $\begin{array}{c}2 \\
(5.1)\end{array}$ & - & - \\
\hline Freedom & $\begin{array}{c}3 \\
(11.1)\end{array}$ & - & $\begin{array}{c}11 \\
(27.5)\end{array}$ & - & $\begin{array}{c}30 \\
(50.8)\end{array}$ & - & $\begin{array}{c}2 \\
(8.0)\end{array}$ & - & $\begin{array}{c}2 \\
(6.7)\end{array}$ & - & $\begin{array}{c}7 \\
(11.9)\end{array}$ & - & $\begin{array}{c}12 \\
(19.7)\end{array}$ & - & $\begin{array}{c}2 \\
(8.7)\end{array}$ & - & $\begin{array}{c}2 \\
(5.1)\end{array}$ & - & - \\
\hline $\begin{array}{c}\text { Getting Better with } \\
\text { Age }\end{array}$ & $\begin{array}{c}0 \\
(00.0)\end{array}$ & - & $\begin{array}{c}5 \\
(12.5)\end{array}$ & - & $\begin{array}{c}0 \\
(00.0)\end{array}$ & - & $\begin{array}{c}6 \\
(24.0)\end{array}$ & - & $\begin{array}{c}4 \\
(13.3)\end{array}$ & - & $\begin{array}{c}9 \\
(15.3)\end{array}$ & - & $\begin{array}{c}5 \\
(8.2)\end{array}$ & - & $\begin{array}{c}2 \\
(8.7)\end{array}$ & - & $\begin{array}{c}1 \\
(2.6)\end{array}$ & - & - \\
\hline Senior Discount & $\begin{array}{c}0 \\
(00.0)\end{array}$ & - & $\begin{array}{c}4 \\
(10.0)\end{array}$ & - & $\begin{array}{c}1 \\
(1.7)\end{array}$ & - & $\begin{array}{c}0 \\
(00.0)\end{array}$ & - & $\begin{array}{c}1 \\
(3.3)\end{array}$ & - & $\begin{array}{c}0 \\
(00.0)\end{array}$ & - & $\begin{array}{c}0 \\
(00.0)\end{array}$ & - & $\begin{array}{c}1 \\
(4.3)\end{array}$ & - & $\begin{array}{c}1 \\
(2.6)\end{array}$ & - & - \\
\hline Respect & $\begin{array}{c}0 \\
(00.0)\end{array}$ & - & $\begin{array}{c}2 \\
(5.0)\end{array}$ & - & $\begin{array}{c}0 \\
(00.0)\end{array}$ & - & $\begin{array}{c}0 \\
(00.0)\end{array}$ & - & $\begin{array}{c}0 \\
(00.0)\end{array}$ & - & $\begin{array}{c}7 \\
(11.9)\end{array}$ & - & $\begin{array}{c}2 \\
(3.3)\end{array}$ & - & $\begin{array}{c}1 \\
(4.3)\end{array}$ & - & $\begin{array}{c}0 \\
(00.0)\end{array}$ & - & - \\
\hline Helping Others & $\begin{array}{c}0 \\
(00.0)\end{array}$ & - & $\begin{array}{c}0 \\
(00.0)\end{array}$ & - & $\begin{array}{c}7 \\
(11.9)\end{array}$ & - & $\begin{array}{c}0 \\
(00.0)\end{array}$ & - & $\begin{array}{c}2 \\
(6.7)\end{array}$ & - & $\begin{array}{c}2 \\
(3.4)\end{array}$ & - & $\begin{array}{c}0 \\
(00.0)\end{array}$ & - & $\begin{array}{c}0 \\
(00.0)\end{array}$ & - & $\begin{array}{c}1 \\
(2.6)\end{array}$ & - & - \\
\hline \multicolumn{20}{|c|}{ Dislikes About Age } \\
\hline Health & $\begin{array}{c}15 \\
(55.6)\end{array}$ & 1.2 & $\begin{array}{c}21 \\
(52.5)\end{array}$ & 4.4 & $\begin{array}{c}28 \\
(47.5)\end{array}$ & 4.7 & $\begin{array}{c}16 \\
(64.0)\end{array}$ & 5.6 & $\begin{array}{c}23 \\
(76.7)\end{array}$ & 6.3 & $\begin{array}{c}26 \\
(44.1)\end{array}$ & 8.3 & $\begin{array}{c}29 \\
(47.5)\end{array}$ & 8.8 & $\begin{array}{c}16 \\
(69.6)\end{array}$ & 5.3 & $\begin{array}{c}13 \\
(33.3)\end{array}$ & 6 & $0.001^{* * *}$ \\
\hline Being Alone & $\begin{array}{c}4 \\
(14.8)\end{array}$ & - & $\begin{array}{c}5 \\
(12.5)\end{array}$ & - & $\begin{array}{c}2 \\
(3.4)\end{array}$ & - & $\begin{array}{c}0 \\
(00.0)\end{array}$ & - & $\begin{array}{c}0 \\
(00.0)\end{array}$ & - & $\begin{array}{c}7 \\
(11.9)\end{array}$ & - & $\begin{array}{c}13 \\
(21.3)\end{array}$ & - & $\begin{array}{c}0 \\
(00.0)\end{array}$ & & $\begin{array}{c}2 \\
(5.1)\end{array}$ & - & - \\
\hline Fear of Death & $\begin{array}{c}1 \\
(3.7)\end{array}$ & - & $\begin{array}{c}6 \\
(15.0)\end{array}$ & - & $\begin{array}{c}1 \\
(1.7)\end{array}$ & - & $\begin{array}{c}1 \\
(4.0)\end{array}$ & - & $\begin{array}{c}0 \\
(00.0)\end{array}$ & - & $\begin{array}{c}0 \\
(00.0)\end{array}$ & - & $\begin{array}{c}1 \\
(1.6)\end{array}$ & - & $\begin{array}{c}1 \\
(4.3)\end{array}$ & - & $\begin{array}{c}0 \\
(00.0)\end{array}$ & - & - \\
\hline Changing World & $\begin{array}{c}5 \\
(18.5)\end{array}$ & $\cdot$ & $\begin{array}{c}0 \\
(00.0)\end{array}$ & - & $\begin{array}{c}1 \\
(1.7)\end{array}$ & - & $\begin{array}{c}0 \\
(00.0)\end{array}$ & - & $\begin{array}{c}1 \\
(3.3)\end{array}$ & - & $\begin{array}{c}3 \\
(5.1)\end{array}$ & - & $\begin{array}{c}3 \\
(4.9)\end{array}$ & - & $\begin{array}{c}0 \\
(00.0)\end{array}$ & - & $\begin{array}{c}0 \\
(00.0)\end{array}$ & - & - \\
\hline Loss of Functionality & $\begin{array}{c}0 \\
(00.0)\end{array}$ & - & $\begin{array}{c}1 \\
(2.5)\end{array}$ & - & $\begin{array}{c}5 \\
(8.5)\end{array}$ & - & $\begin{array}{c}0 \\
(00.0)\end{array}$ & - & $\begin{array}{c}0 \\
(00.0)\end{array}$ & 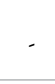 & $\begin{array}{c}0 \\
(00.0)\end{array}$ & - & $\begin{array}{c}1 \\
(1.6)\end{array}$ & . & $\begin{array}{c}0 \\
(00.0)\end{array}$ & - & $\begin{array}{c}0 \\
(00.0)\end{array}$ & - & - \\
\hline $\begin{array}{l}\text { Loss Financial } \\
\text { Independence }\end{array}$ & $\begin{array}{c}0 \\
(00.0)\end{array}$ & - & $\begin{array}{c}0 \\
(00.0)\end{array}$ & - & $\begin{array}{c}5 \\
(8.5)\end{array}$ & - & $\begin{array}{c}0 \\
(00.0)\end{array}$ & - & $\begin{array}{c}0 \\
(00.0)\end{array}$ & - & $\begin{array}{c}0 \\
(00.0)\end{array}$ & - & $\begin{array}{c}0 \\
(00.0)\end{array}$ & - & $\begin{array}{c}1 \\
(4.3)\end{array}$ & . & $\begin{array}{c}0 \\
(00.0)\end{array}$ & - & - \\
\hline Dependency & $\begin{array}{c}0 \\
(00.0)\end{array}$ & - & $\begin{array}{c}0 \\
(00.0)\end{array}$ & . & $\begin{array}{c}0 \\
(00.0)\end{array}$ & . & $\begin{array}{c}0 \\
(00.0)\end{array}$ & - & $\begin{array}{c}1 \\
(3.3)\end{array}$ & - & $\begin{array}{c}1 \\
(1.7)\end{array}$ & . & $\begin{array}{c}9 \\
(14.8)\end{array}$ & . & $\begin{array}{c}0 \\
(00.0)\end{array}$ & - & $\begin{array}{c}0 \\
(00.0)\end{array}$ & - & - \\
\hline Nothing & $\begin{array}{c}0 \\
(00.0)\end{array}$ & - & $\begin{array}{c}1 \\
(2.5)\end{array}$ & . & $\begin{array}{c}1 \\
(1.7)\end{array}$ & . & $\begin{array}{c}0 \\
(00.0)\end{array}$ & - & $\begin{array}{c}2 \\
(6.7)\end{array}$ & . & $\begin{array}{c}11 \\
(18.6)\end{array}$ & - & $\begin{array}{c}1 \\
(1.6)\end{array}$ & . & $\begin{array}{c}4 \\
(17.4)\end{array}$ & . & $\begin{array}{c}17 \\
(43.6)\end{array}$ & - & - \\
\hline Working & $\begin{array}{c}0 \\
(00.0)\end{array}$ & - & $\begin{array}{c}0 \\
(00.0)\end{array}$ & - & $\begin{array}{c}3 \\
(5.1)\end{array}$ & . & $\begin{array}{c}3 \\
(12.0)\end{array}$ & - & $\begin{array}{c}0 \\
(00.0)\end{array}$ & . & $\begin{array}{c}0 \\
(00.0)\end{array}$ & - & $\begin{array}{c}0 \\
(00.0)\end{array}$ & . & $\begin{array}{c}1 \\
(4.3)\end{array}$ & - & $\begin{array}{c}0 \\
(00.0)\end{array}$ & - & - \\
\hline $\begin{array}{l}\text { Being Called Old/No } \\
\text { Respect }\end{array}$ & $\begin{array}{c}0 \\
(00.0)\end{array}$ & - & $\begin{array}{c}2 \\
(5.0)\end{array}$ & - & $\begin{array}{c}1 \\
(1.7)\end{array}$ & - & $\begin{array}{c}0 \\
(00.0)\end{array}$ & - & $\begin{array}{c}3 \\
(10.0)\end{array}$ & 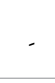 & $\begin{array}{c}2 \\
(3.4)\end{array}$ & - & $\begin{array}{c}2 \\
(3.3)\end{array}$ & - & $\begin{array}{c}0 \\
(00.0)\end{array}$ & - & $\begin{array}{c}0 \\
(00.0)\end{array}$ & - & - \\
\hline Everything & $\begin{array}{c}0 \\
(00.0)\end{array}$ & - & $\begin{array}{c}1 \\
(2.5)\end{array}$ & - & $\begin{array}{c}0 \\
(00.0)\end{array}$ & . & $\begin{array}{c}2 \\
(8.0)\end{array}$ & - & $\begin{array}{c}0 \\
(00.0)\end{array}$ & 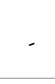 & $\begin{array}{c}0 \\
(00.0)\end{array}$ & - & $\begin{array}{c}0 \\
(00.0)\end{array}$ & - & $\begin{array}{c}0 \\
(00.0)\end{array}$ & - & $\begin{array}{c}0 \\
(00.0)\end{array}$ & - & - \\
\hline Accepting Aging & $\begin{array}{c}0 \\
(00.0)\end{array}$ & . & $\begin{array}{c}0 \\
(00.0)\end{array}$ & . & $\begin{array}{c}0 \\
(00.0)\end{array}$ & . & $\begin{array}{c}0 \\
(00.0)\end{array}$ & . & $\begin{array}{c}0 \\
(00.0)\end{array}$ & 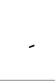 & $\begin{array}{c}5 \\
(8.5)\end{array}$ & - & $\begin{array}{c}2 \\
(3.3)\end{array}$ & - & $\begin{array}{c}0 \\
(00.0)\end{array}$ & - & $\begin{array}{c}0 \\
(00.0)\end{array}$ & - & - \\
\hline No Response & $\begin{array}{c}2 \\
(7.4)\end{array}$ & - & $\begin{array}{c}3 \\
(7.5)\end{array}$ & - & $\begin{array}{c}12 \\
(20.3)\end{array}$ & . & $\begin{array}{c}3 \\
(12.0)\end{array}$ & . & $\begin{array}{c}0 \\
(00.0)\end{array}$ & . & $\begin{array}{c}4 \\
(6.8)\end{array}$ & . & $\begin{array}{c}0 \\
(00.0)\end{array}$ & . & $\begin{array}{c}0 \\
(00.0)\end{array}$ & - & $\begin{array}{c}7 \\
(17.9)\end{array}$ & - & - \\
\hline \multicolumn{20}{|c|}{ Looking Forward To } \\
\hline Travel & $\begin{array}{c}2 \\
(7.4)\end{array}$ & 4.2 & $\begin{array}{c}13 \\
(32.5)\end{array}$ & 8.1 & $\begin{array}{c}0 \\
(00.0)\end{array}$ & 4.6 & $\begin{array}{c}0 \\
(00.0)\end{array}$ & 3.9 & $\begin{array}{c}2 \\
(6.7)\end{array}$ & 6.7 & $\begin{array}{c}0 \\
(00.0)\end{array}$ & 9.8 & $\begin{array}{c}7 \\
(11.5)\end{array}$ & 11.2 & $\begin{array}{c}5 \\
(21.7)\end{array}$ & 3.7 & $\begin{array}{c}6 \\
(15.4)\end{array}$ & 3.7 & $0.001^{* * *}$ \\
\hline Peaceful Death & $\begin{array}{c}1 \\
(3.7)\end{array}$ & - & $\begin{array}{c}2 \\
(5.0)\end{array}$ & 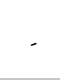 & $\begin{array}{c}2 \\
(3.4)\end{array}$ & - & $\begin{array}{c}16 \\
(64.0)\end{array}$ & - & $\begin{array}{c}0 \\
(00.0)\end{array}$ & - & $\begin{array}{c}11 \\
(18.6)\end{array}$ & - & $\begin{array}{c}1 \\
(1.6)\end{array}$ & - & $\begin{array}{c}0 \\
(00.0)\end{array}$ & & $\begin{array}{c}0 \\
(00.0)\end{array}$ & - & - \\
\hline Surviving & $\begin{array}{c}2 \\
(7.4)\end{array}$ & - & $\begin{array}{c}1 \\
(2.5)\end{array}$ & - & $\begin{array}{c}8 \\
(13.6)\end{array}$ & - & $\begin{array}{c}2 \\
(8.0)\end{array}$ & 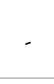 & $\begin{array}{c}5 \\
(16.7)\end{array}$ & - & $\begin{array}{c}4 \\
(6.8)\end{array}$ & - & $\begin{array}{c}2 \\
(3.3)\end{array}$ & - & $\begin{array}{c}0 \\
(00.0)\end{array}$ & - & $\begin{array}{c}3 \\
(7.7)\end{array}$ & - & - \\
\hline Activities/Reading & $\begin{array}{c}5 \\
(18.5)\end{array}$ & - & $\begin{array}{c}1 \\
(2.5)\end{array}$ & - & $\begin{array}{c}2 \\
(3.4)\end{array}$ & - & $\begin{array}{c}0 \\
(00.0)\end{array}$ & - & $\begin{array}{c}2 \\
(6.7)\end{array}$ & - & $\begin{array}{c}4 \\
(6.8)\end{array}$ & - & $\begin{array}{c}1 \\
(1.6)\end{array}$ & - & $\begin{array}{c}6 \\
(26.1)\end{array}$ & - & $\begin{array}{c}2 \\
(5.1)\end{array}$ & - & - \\
\hline Being with Family & $\begin{array}{c}6 \\
(22.2)\end{array}$ & - & $\begin{array}{c}16 \\
(40.0)\end{array}$ & - & $\begin{array}{c}15 \\
(25.4)\end{array}$ & - & $\begin{array}{c}2 \\
(8.0)\end{array}$ & - & $\begin{array}{c}11 \\
(36.7)\end{array}$ & - & $\begin{array}{c}11 \\
(18.6)\end{array}$ & . & $\begin{array}{c}23 \\
(37.7)\end{array}$ & - & $\begin{array}{c}8 \\
(34.8)\end{array}$ & - & $\begin{array}{c}6 \\
(15.4)\end{array}$ & - & - \\
\hline Improving Health & $\begin{array}{c}1 \\
(3.7)\end{array}$ & - & $\begin{array}{c}0 \\
(00.0)\end{array}$ & - & $\begin{array}{c}17 \\
(28.8)\end{array}$ & . & $\begin{array}{c}0 \\
(00.0)\end{array}$ & - & $\begin{array}{c}6 \\
(20.0)\end{array}$ & - & $\begin{array}{c}10 \\
(16.9)\end{array}$ & - & $\begin{array}{c}4 \\
(6.6)\end{array}$ & . & $\begin{array}{c}0 \\
(00.0)\end{array}$ & - & $\begin{array}{c}12 \\
(30.8)\end{array}$ & - & - \\
\hline $\begin{array}{l}\text { Winning Lottery/ } \\
\text { Money }\end{array}$ & $\begin{array}{c}1 \\
(3.7)\end{array}$ & - & $\begin{array}{c}0 \\
(00.0)\end{array}$ & - & $\begin{array}{c}4 \\
(6.8)\end{array}$ & . & $\begin{array}{c}1 \\
(4.0)\end{array}$ & - & $\begin{array}{c}0 \\
(00.0)\end{array}$ & - & $\begin{array}{c}5 \\
(8.5)\end{array}$ & - & $\begin{array}{c}2 \\
(3.3)\end{array}$ & . & $\begin{array}{c}0 \\
(00.0)\end{array}$ & - & $\begin{array}{c}5 \\
(12.8)\end{array}$ & - & - \\
\hline Nothing & $\begin{array}{c}6 \\
(22.2)\end{array}$ & - & $\begin{array}{c}1 \\
(2.5)\end{array}$ & - & $\begin{array}{c}5 \\
(8.5)\end{array}$ & . & $\begin{array}{c}0 \\
(00.0)\end{array}$ &. & $\begin{array}{c}1 \\
(3.3)\end{array}$ & - & $\begin{array}{c}1 \\
(1.7)\end{array}$ & - & $\begin{array}{c}4 \\
(6.6)\end{array}$ & . & $\begin{array}{c}2 \\
(8.7)\end{array}$ & . & $\begin{array}{c}0 \\
(00.0)\end{array}$ & . & . \\
\hline Working & $\begin{array}{c}0 \\
(00.0)\end{array}$ & - & $\begin{array}{c}1 \\
(2.5)\end{array}$ & - & $\begin{array}{c}1 \\
(1.7)\end{array}$ & - & $\begin{array}{c}2 \\
(8.0)\end{array}$ & . & $\begin{array}{c}0 \\
(00.0)\end{array}$ & 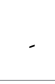 & $\begin{array}{c}0 \\
(00.0)\end{array}$ & . & $\begin{array}{c}0 \\
(00.0)\end{array}$ & . & $\begin{array}{c}0 \\
(00.0)\end{array}$ & - & $\begin{array}{c}0 \\
(00.0)\end{array}$ & - & - \\
\hline Retirement & $\begin{array}{c}0 \\
(00.0)\end{array}$ & - & $\begin{array}{c}5 \\
(12.5)\end{array}$ & - & $\begin{array}{c}0 \\
(00.0)\end{array}$ & - & $\begin{array}{c}0 \\
(00.0)\end{array}$ & - & $\begin{array}{c}0 \\
(00.0)\end{array}$ & - & $\begin{array}{c}0 \\
(00.0)\end{array}$ & . & $\begin{array}{c}1 \\
(1.6)\end{array}$ & . & $\begin{array}{c}0 \\
(00.0)\end{array}$ & . & $\begin{array}{c}0 \\
(00.0)\end{array}$ & - & - \\
\hline
\end{tabular}




\begin{tabular}{|c|c|c|c|c|c|c|c|c|c|c|c|c|c|c|c|c|c|c|c|}
\hline $\begin{array}{l}\text { Setting Good } \\
\text { Example }\end{array}$ & $\begin{array}{c}0 \\
(00.0)\end{array}$ & & $\begin{array}{c}0 \\
(00.0)\end{array}$ & - & $\begin{array}{c}2 \\
(3.4)\end{array}$ & - & $\begin{array}{c}0 \\
(00.0)\end{array}$ & - & $\begin{array}{c}0 \\
(00.0)\end{array}$ & & $\begin{array}{c}0 \\
(00.0)\end{array}$ & & $\begin{array}{c}0 \\
(00.0)\end{array}$ & - & $\begin{array}{c}0 \\
(00.0)\end{array}$ & - & $\begin{array}{c}0 \\
(00.0)\end{array}$ & - & - \\
\hline Not Being a Burden & $\begin{array}{c}0 \\
(00.0)\end{array}$ & - & $\begin{array}{c}0 \\
(00.0)\end{array}$ & - & $\begin{array}{c}2 \\
(3.4)\end{array}$ & - & $\begin{array}{c}0 \\
(00.0)\end{array}$ & - & $\begin{array}{c}0 \\
(00.0)\end{array}$ & - & $\begin{array}{c}0 \\
(00.0)\end{array}$ & - & $\begin{array}{c}0 \\
(00.0)\end{array}$ & - & $\begin{array}{c}0 \\
(00.0)\end{array}$ & - & $\begin{array}{c}0 \\
(00.0)\end{array}$ & - & - \\
\hline Practicing My Faith & $\begin{array}{c}0 \\
(00.0)\end{array}$ & - & $\begin{array}{c}0 \\
(00.0)\end{array}$ & - & $\begin{array}{c}0 \\
(00.0)\end{array}$ & - & $\begin{array}{c}0 \\
(00.0)\end{array}$ & - & $\begin{array}{c}2 \\
(6.7)\end{array}$ & - & $\begin{array}{c}11 \\
(18.6)\end{array}$ & - & $\begin{array}{c}16 \\
(26.2)\end{array}$ & - & $\begin{array}{c}0 \\
(00.0)\end{array}$ & - & $\begin{array}{c}0 \\
(00.0)\end{array}$ & - & - \\
\hline \multicolumn{20}{|c|}{ Advice for the Young } \\
\hline $\begin{array}{l}\text { Focus on Work and } \\
\text { Family }\end{array}$ & $\begin{array}{c}11 \\
(40.7)\end{array}$ & 2.5 & $\begin{array}{c}2 \\
(5.0)\end{array}$ & 3.5 & $\begin{array}{c}3 \\
(5.1)\end{array}$ & 4.6 & $\begin{array}{c}0 \\
(00.0)\end{array}$ & 5.5 & $\begin{array}{c}3 \\
(10.0)\end{array}$ & 5.9 & $\begin{array}{c}6 \\
(10.2)\end{array}$ & 6.5 & $\begin{array}{c}14 \\
(23.0)\end{array}$ & 5.2 & $\begin{array}{c}5 \\
(21.7)\end{array}$ & 5.8 & $\begin{array}{c}7 \\
(17.9)\end{array}$ & 5.5 & $0.001^{* * *}$ \\
\hline Listen to Elders & $\begin{array}{c}2 \\
(7.4)\end{array}$ & - & $\begin{array}{c}0 \\
(00.0)\end{array}$ & - & $\begin{array}{c}0 \\
(00.0)\end{array}$ & - & $\begin{array}{c}0 \\
(00.0)\end{array}$ & 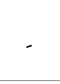 & $\begin{array}{c}12 \\
(40.0)\end{array}$ & - & $\begin{array}{c}1 \\
(1.7)\end{array}$ & - & $\begin{array}{c}8 \\
(13.1)\end{array}$ & - & $\begin{array}{c}0 \\
(00.0)\end{array}$ & - & $\begin{array}{c}0 \\
(00.0)\end{array}$ & - & - \\
\hline Be Honest & $\begin{array}{c}5 \\
(18.5)\end{array}$ & - & $\begin{array}{c}10 \\
(25.0)\end{array}$ & - & $\begin{array}{c}9 \\
(15.3)\end{array}$ & - & $\begin{array}{c}0 \\
(00.0)\end{array}$ & - & $\begin{array}{c}1 \\
(3.3)\end{array}$ & - & $\begin{array}{c}4 \\
(6.8)\end{array}$ & - & $\begin{array}{c}9 \\
(14.8)\end{array}$ & - & $\begin{array}{c}3 \\
(13.0)\end{array}$ & - & $\begin{array}{c}9 \\
(23.1)\end{array}$ & - & - \\
\hline Save Money & $\begin{array}{c}1 \\
(3.7)\end{array}$ & - & $\begin{array}{c}3 \\
(7.5)\end{array}$ & - & $\begin{array}{c}11 \\
(18.6)\end{array}$ & - & $\begin{array}{c}2 \\
(8.0)\end{array}$ & - & $\begin{array}{c}2 \\
(6.7)\end{array}$ & - & $\begin{array}{c}1 \\
(1.7)\end{array}$ & - & $\begin{array}{c}6 \\
(9.8)\end{array}$ & - & $\begin{array}{c}2 \\
(8.7)\end{array}$ & - & $\begin{array}{c}2 \\
(5.1)\end{array}$ & - & - \\
\hline Get an Education & $\begin{array}{c}2 \\
(7.4)\end{array}$ & - & $\begin{array}{c}2 \\
(5.0)\end{array}$ & - & $\begin{array}{c}1 \\
(1.7)\end{array}$ & - & $\begin{array}{c}0 \\
(00.0)\end{array}$ & - & $\begin{array}{c}1 \\
(3.3)\end{array}$ & - & $\begin{array}{c}4 \\
(6.8)\end{array}$ & - & $\begin{array}{c}4 \\
(6.6)\end{array}$ & - & $\begin{array}{c}5 \\
(21.7)\end{array}$ & - & $\begin{array}{c}3 \\
(7.7)\end{array}$ & - & - \\
\hline Don't Do Drugs & $\begin{array}{c}1 \\
(3.7)\end{array}$ & - & $\begin{array}{c}2 \\
(5.0)\end{array}$ & - & $\begin{array}{c}0 \\
(00.0)\end{array}$ & - & $\begin{array}{c}0 \\
(00.0)\end{array}$ & - & $\begin{array}{c}1 \\
(3.3)\end{array}$ & - & $\begin{array}{c}1 \\
(1.7)\end{array}$ & - & $\begin{array}{c}1 \\
(1.6)\end{array}$ & - & $\begin{array}{c}0 \\
(00.0)\end{array}$ & - & $\begin{array}{c}1 \\
(2.6)\end{array}$ & - & - \\
\hline Live to the Fullest & $\begin{array}{c}1 \\
(3.7)\end{array}$ & - & $\begin{array}{c}16 \\
(40.0)\end{array}$ & - & $\begin{array}{c}7 \\
(11.9)\end{array}$ & - & $\begin{array}{c}0 \\
(00.0)\end{array}$ & - & $\begin{array}{c}3 \\
(10.0)\end{array}$ & - & $\begin{array}{c}11 \\
(18.6)\end{array}$ & - & $\begin{array}{c}9 \\
(14.8)\end{array}$ & - & $\begin{array}{c}0 \\
(00.0)\end{array}$ & - & $\begin{array}{c}2 \\
(5.1)\end{array}$ & - & - \\
\hline Be Healthy/Exercise & $\begin{array}{c}0 \\
(00.0)\end{array}$ & - & $\begin{array}{c}4 \\
(10.0)\end{array}$ & - & $\begin{array}{c}26 \\
(44.1)\end{array}$ & - & $\begin{array}{c}2 \\
(8.0)\end{array}$ & - & $\begin{array}{c}3 \\
(10.0)\end{array}$ & - & $\begin{array}{c}10 \\
(16.9)\end{array}$ & - & $\begin{array}{c}9 \\
(14.8)\end{array}$ & - & $\begin{array}{c}3 \\
(13.0)\end{array}$ & - & $\begin{array}{c}11 \\
(28.2)\end{array}$ & - & - \\
\hline Believe in God/Allah & $\begin{array}{c}0 \\
(00.0)\end{array}$ & - & $\begin{array}{c}0 \\
(00.0)\end{array}$ & - & $\begin{array}{c}0 \\
(00.0)\end{array}$ & - & $\begin{array}{c}1 \\
(4.0)\end{array}$ & - & $\begin{array}{c}4 \\
(13.3)\end{array}$ & - & $\begin{array}{c}16 \\
(27.1)\end{array}$ & - & $\begin{array}{c}0 \\
(00.0)\end{array}$ & - & $\begin{array}{c}0 \\
(00.0)\end{array}$ & - & $\begin{array}{c}0 \\
(00.0)\end{array}$ & - & - \\
\hline Get Married & $\begin{array}{c}0 \\
(00.0)\end{array}$ & - & $\begin{array}{c}0 \\
(00.0)\end{array}$ & . & $\begin{array}{c}0 \\
(00.0)\end{array}$ & - & $\begin{array}{c}1 \\
(4.0)\end{array}$ & - & $\begin{array}{c}0 \\
(00.0)\end{array}$ & - & $\begin{array}{c}2 \\
(3.4)\end{array}$ & - & $\begin{array}{c}0 \\
(00.0)\end{array}$ & - & $\begin{array}{c}2 \\
(8.7)\end{array}$ & - & $\begin{array}{c}2 \\
(5.1)\end{array}$ & - & - \\
\hline $\begin{array}{l}\text { Don't Depend on } \\
\text { Gov't }\end{array}$ & $\begin{array}{c}0 \\
(00.0)\end{array}$ & - & $\begin{array}{c}0 \\
(00.0)\end{array}$ & - & $\begin{array}{c}0 \\
(00.0)\end{array}$ & - & $\begin{array}{c}0 \\
(00.0)\end{array}$ & - & $\begin{array}{c}0 \\
(00.0)\end{array}$ & 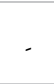 & $\begin{array}{c}1 \\
(1.7)\end{array}$ & . & $\begin{array}{c}1 \\
(1.6)\end{array}$ & - & $\begin{array}{c}0 \\
(00.0)\end{array}$ & - & $\begin{array}{c}0 \\
(00.0)\end{array}$ & - & - \\
\hline
\end{tabular}

Note: Percentages that do not add up to 100 are the result of no response; Participants were asked to select what was the most important aspect for each question; */***** indicate significant differences at .05/.01/.001 levels based on group mean comparisons between samples

respondents had retirement benefits, and feelings about current age. There was no evidence of significant effects ( $>>0.17)$. We also examined cultural component with whether retirement benefits are sufficient. Respondents from collectivist cultures reported retirement benefits were sufficient more $(\mathrm{M}=0.77, \mathrm{SD}=0.85)$ than those living in individualistic countries $(\mathrm{M}=0.54, \mathrm{SD}=0.82)$ [F (1, 282) $=4.48, \mathrm{p}<0.03]$.

\section{DISCUSSION}

The principle goal for this study was to evaluate older adults' aging experiences in multiple countries ass literature suggests etic (universal) and emic (culturally specific) elements exist in the aging experience worldwide. Much of the prior data has been from a single location and, in some instances, has led to an overgeneralization in the description of human aging. Interviews with 363 participants yielded several relevant findings with regard to aging across the participants from these nine specific countries. First, those with the highest number of reported activities indicated less satisfaction with life and more negative feelings about being their current age. Of particular interest was that daily activity levels were not related to self-report of being happy and healthy. Second, older adults reporting being happy and healthy reported a lower overall satisfaction with life and were not receiving retirement benefits at the time of interview. Third, individuals who stated not being very satisfied with life also indicate their retirement benefits as not being sufficient.

\section{Cultural diversity of the aging experience}

Cultural understanding has become an even more important topic as researchers, policy makers, and the general public seeks to understand the similarities and variations found within and between populations. As reported by previous research [24] current study participants demonstrated variation for the variables of interest. Significant differences were found for level of being happy and health, overall satisfaction with life, the reporting of retirement benefits and source of benefits, retirement benefit sufficiency, the most important benefit of their age, what is disliked about being this age, what respondents by cohort were looking forward to and the advice given to youth. Controlling for gender and age produced no change on the diversity of response for any of the variables of interest.

\section{Cultural uniformity of the aging experience}

Data collected from older adults from these nine countries investigated suggest the attitudes and behaviors of aging adults differ in significant ways. However, uniformity of response was suggested with health being the factor most identified as the thing participants said they disliked about getting older but spending time with family was what they looked most forward to as they continue to age. Current study data suggest etic/universal and emic/culturespecific factors exist with reference to the aging experience. These findings are consistent with previous studies examining aging using a cultural lens $[20,21,22]$. This study adds to the body of work about how cultural similarity in various aspects of aging and the associations between factors are consistent between eastern and western countries [37] and as reported by [27], the present data also suggests older adults have similar concerns about aging, but not all have the exact same concern. 
Methodologies that explore age-related similarities and differences across countries are emergent $[1,4,38,39]$. Research must also be adaptive to include the experience aging in an ever-increasingly diverse world. An in-depth knowledge of the aging experience in many places can only make our awareness of the life course more complete [40].

\section{LIMITATIONS}

Limitations for the study should be noted. First, snowball sampling relegates the participant pool to investigator access. As these samples were not representative, so our results should not be overgeneralized. Unequal numbers of male and female participants, dissimilar age means, and urban versus rural places of birth may have influenced some of the variation reported between the groups. Second, collection of data from various countries creates in and of itself unexpected challenges (i.e., translation of surveys, preparation toward consistent data collection protocols, obtaining of similar participant demographic characteristics).

\section{CONCLUSION}

The results of the current research efforts were intended to understand culturally-based aging perceptions. With the data collected, we sought to develop a typology toward the understanding of features supporting cultural uniformity and diverse components of aging. There was limited consistency in variables used in the literature in trying to understand the aging experience within a culture specific framework. As a result, there is a need to develop an aging framework stemming from cross-national data collection, which should be the norm for published social science research.

One tangible step in developing a framework could be the creation of an aging perception measure. Such an instrument might be translated for use in all countries so as to further investigate aging across the globe. Although development of a questionnaire that would effectively measure the aging experience across cultures might be difficult, pairing variables that are uniform no matter the location along-side diverse factors could produce a clearer picture of what it means to age in place. As a result of the data collected, a slice of cultural understanding was gained as we know more about each aging in each county. Future research needs to employ cultural humility to further explore aging in global settings.

\section{ACKNOWLEDGEMENT}

The authors would like to thank Narumon Saratapun, Suportip Pupaneau, Keinyatse Kgosidialwa, Wejdan Felmban, Katherine Sammons, Akiko Watake, Maha Ben Salem, Zixi Lyu for their assistance with this eight-year project.

\section{REFERENCES}

1. http://etheses.whiterose.ac.uk/4213/1/425593.pdf.

2. Bilotta C, Bowling A, Casè A, Nicolini P, Mauri S, Castelli M, et al. Dimensions and correlates of quality of life according to frailty status: A cross-section study on community-dwelling older adults referred to an outpatient geriatric service in Italy. Health Qual Life Outcomes 2010;8:56-65.

3. Gray RS, Chamratrithirong A. The international handbook on aging: Thailand. Denver: Praeger. 2009.

4. Kincannon CL, He W, West LA. Demography of aging in
China and the United States and the economic well-being of their older populations. J Cross Cult Psycho 2005;20(3):243-255.

5. Malhotra R, Bautista MAC, Muller AM, Koh GCH, Theng Y, Hoskins SJ, et al. The aging of a young nation: Population aging in Singapore. Gerontologist 2018;58(7): 1-11.

6. Karlin NJ, Ben Salem M, Weil J. Aging in Tunisia. Gerontologist, 2018; 58(6):1004-1008.

7. http://www.un.org/swaa2002/coverage/vforumE.htm

8. Crowther M, Austin A. The cultural context of clinical work with aging caregivers. In: Qualls SH, Zarit SH (eds.) Aging Families and Caregiving. Hoboken, New Jersey: John Wiley \& Sons Inc., USA, 2009;45-60.

9. Hiroto KE, Yarry SJ. Cultural differences in aging experiences of ethnic and sexual minority older adults. In: Yochim B, Woodhead E (eds.) Psychology of aging: A biopsychosocial perspective. New York : Springer Publishing Company LLC, USA. 2017:299-322.

10. Iwasaki M, Tazeau YN, Kimmel D, Baker NL, McCallum TJ. Praeger perspectives: Race and ethnicity in psychology. In: Chin JL (eds.) Diversity in Mind and in Action: Vol. 3. Social justice matters. Santa Barbara, CA, US: Praeger/ABC-CLIO, XVIII, 226;2009:71-90.

11. Karlin NJ, Weil J, Felmban W. Aging in Saudi Arabia: An exploratory study of contemporary older persons' views about daily life, health, and the experience of aging. Gerontol Geriatric Med 2016;2:1-9.

12. Karlin NJ, Weil J, Sammons K. Semi-structured interviewing of older adults in seven countries. In: Maurer C (eds.) SAGE Research Methods Cases-Part 2. SAGE Publications Ltd London, England, UK, 2017.

13. Tazeau Y. Individual and cultural diversity considerations in geropsychology. In: Molinari V (eds). Specialty Competencies in Geropsychology New York: Oxford University Press; 2011:103-114.

14. Weil J, Karlin NJ, Floren K. Boomer backlash as aging becomes a global institution. Int J Contemp Sociol 2015;52(1):37-58.

15. Chen X, Silverstein M. Intergenerational social support and the psychological well-being of older parents in China. Aging 2000;22(1):43-65.

16. Penning MJ, Cloutier DS, Nuernberger K, MacDonald SWS, Taylor D. Long-term care trajectories in Canadian context: Patterns and predictors of publicly funded care. J Gerontol B Psychol Sci 2018;73:1077-1087.

17. Schatz E, Ralston M, Madhavan S, Collinson MA, GomezOlive FX. Living arrangements, disability and gender of older adults among rural South Africa. J Gerontol B Psychol Sci 2018;73(6):1112-1122.

18. Song Y, Sorensen S, Yan ECW. Family support and preparation for future care needs among urban Chinese baby boomers. J Gerontol B Psychol Sci 2018;73(6):1066-76.

19. Sun R. Old age support in contemporary urban China from both parents' and children's perspectives. Res Aging 2002;24(3):337-359.

20. Angelini V, Howdon DDH, Mierau JO. Childhood socioeconomic status and late-adulthood mental health: Results from the Survey on Health, Ageing, and Retirement in Europe. J Gerontol B Psychol Sci Soc Sci 2019;74(1):95-104. 
21. Ladin K, Reinhold S. Health of aging immigrants and native born men across 11 European countries. J Gerontol B Psychol Sci 2013;68(2):298-309.

22. Li T, Fung HH. Age differences in trust: An investigation across 38 countries. J Gerontol B Psychol Sci Soc Sci. 2013;68(3):347-355.

23. Yun RJ, Lachman ME. Perceptions of aging in two cultures: Korean and American views on old age. J Cross Cult Gerontol 2006;21:55-70.

24. Allik J, McCrae RR. Toward a geography of personality traits: Patterns of profiles across 36 cultures. J Cross Cult Psycho 2004;35(1):13-28.

25. Yount K. Gender and intergenerational co-residence in Egypt and Tunisia. Popul Res Policy Rev 2009;28(5):615-640.

26. Yi Z, Vaupel JW. Functional capacity and self-evaluation of health and life of oldest old in China. J Soc Issues 2002;58:733-738.

27. Oksuzyan A, Singh PK, Christensen K, Jasilionis D. A crossnational study of the gender gap in health among older adults in India and China: Similarities and disparities. Gerontologist 2018;58(6):1156-1165.

28. Kornadt AE, Voss P, Fung HH, Hess TM, Rothermund K. Preparation of old age: The role of cultural context and future perceptions. J Gerontol B Psychol Sci Soc Sci 2019; 74(4):609-619.

29. Fiske S. Social being: Core motives in social psychology. Hoboken, New Jersey: John Wiley \& Sons, Inc., USA, 2018.

30. Triandis HC, Bontempo R, Villareal MJ, Asai M, Lucca N.
Individualism and collectivism: Cross-cultural perspectives on self-ingroup relationships.J Pers Soc Psychol.1988;54(2):323-338.

31. Kashima Y, Yamaguchi S, Kim U, Choi SC, Gelfand MJ, Yuki M. Culture, gender, and self: A perspective from individualismcollectivism research. J Pers Soc Psychol 1995;69(5):925-937.

32. Mead M. Cooperation and competition among primitive people. Boston: Beacon, 1967.

33. Mesquita B. Emotions in collectivist and individualist contexts. J Pers Soc Psychol 2001;80(1): 68-74.

34. Oyserman D, Lee SWS. Does culture influence what and how we think? Effects of priming individualism and collectivism. Psychol Bull 2008;134 (2):311-342.

35. Schwarz N. Individualism and collectivism. J Consum Psychol 2006;16(4):324

36. Wacker RR, Roberto KA. Aging social policies: An international perspective. Los Angeles, CA: Sage, 2010.

37. Chan D, Hamamura T, Li LMW, Zhang X. Is trusting others related to better health? An investigation of older adults across six non-western countries. J Cross Cult Psycho 2017; 48(8):1288-1301.

38. Makoni S. Aging in Africa: A critical review. J Cross Cult Gerontol 2008;23(2):199-209.

39. http://cfs.hivci.org/country-factsheet.html

40. Karlin NJ, Weil J. Healthy aging in a global context: Comparing six countries. Ageing Int 2017;42(1):1-22. 